Pacific

Journal of

Mathematics

UNIQUENESS OF WHITTAKER MODEL FOR THE METAPLECTIC GROUP

DANI SZPRUCH

Volume $232 \quad$ No. 2

October 2007 


\title{
UNIQUENESS OF WHITTAKER MODEL FOR THE METAPLECTIC GROUP
}

\author{
DANI SZPRUCH
}

Let $F$ be a local nonarchimedean field. We prove the uniqueness of the Whittaker model for irreducible admissible representations of $\overline{\operatorname{Sp}_{2 n}(F)}$, the metaplectic double cover of $\operatorname{Sp}_{2 n}(F)$. An ingredient of the proof is an explicit extension of Rao's normalized cocycle from $\operatorname{Sp}_{2 n}(F)$ to $\operatorname{GSp}_{2 n}(F)$.

\section{Introduction}

Let $F$ be a $p$-adic field. Let $\overline{\operatorname{Sp}_{2 n}(F)}$ be the metaplectic double cover of $\operatorname{Sp}_{2 n}(F)$. The uniqueness of Whittaker models for irreducible admissible representations of $\overline{\mathrm{Sp}_{2 n}(F)}$ is well known to experts. Although it has been used many times, there is no general written proof, although a uniqueness theorem for principal series representations can be found in [Bump et al. 1991]. In this paper, we correct the situation and prove this uniqueness as Theorem 3.4. It turns out that one may use similar methods to those used in [Shalika 1974; Gel'fand and Kazhdan 1975; Bernstein and Zelevinskii 1976] for quasisplit groups over $F$. A central role is played by $\bar{\tau}$, a certain involution on $\overline{\operatorname{Sp}_{2 n}(F)}$ which is an extension of the involution $g \mapsto{ }^{\tau} g=\sigma_{0}{ }^{\mathrm{t}} g \sigma_{0}^{-1}$ defined on $\operatorname{Sp}_{2 n}(F)$, where $\sigma_{0}$ is a certain Weyl element.

Here, we realize $\overline{\mathrm{Sp}_{2 n}(F)}$ using Rao's cocycle [1993]. In Section 2, we extend it explicitly to $\operatorname{GSp}_{2 n}(F) \times \operatorname{GSp}_{2 n}(F)$ and then use this extension to define $\bar{\tau}$. The unexpected fact, at least to us, is that $\tau$ extends to $\overline{\operatorname{Sp}_{2 n}(F)}$ in the simplest possible way, namely ${ }^{\bar{\tau}}(g, \epsilon)=\left({ }^{\tau} g, \epsilon\right)$.

\section{Rao's cocycle}

Let $F$ be a local nonarchimedean field. Let $X=F^{2 n}$ be a vector space of even dimension over $F$ equipped with $\langle\cdot, \cdot\rangle: X \times X \rightarrow F$, a non degenerate symplectic form, and let $\operatorname{Sp}(X)=\operatorname{Sp}_{2 n}(F)$ be the subgroup of $\mathrm{GL}(X)$ of symplectomorphisms of $X$ onto itself. We shall write the action of $G L(X)$ on $X$ from the right. R. Rao [1993] constructs an explicit nontrivial 2-cocycle $c(\cdot, \cdot)$ on $\operatorname{Sp}(X)$. The

Keywords: Whittaker model, metaplectic group, Rao's cocycle. 
set $\overline{\mathrm{Sp}(X)}=\mathrm{Sp}(X) \times\{ \pm 1\}$ is then made a group, called the metaplectic group, via

$$
\left(g_{1}, \epsilon_{1}\right)\left(g_{2}, \epsilon_{2}\right)=\left(g_{1} g_{2}, \epsilon_{1} \epsilon_{2} c\left(g_{1}, g_{2}\right)\right) .
$$

In Section 2A, we describe this cocycle.

Let $\operatorname{GSp}(X)$ be the group of similitudes of the symplectic form above. It is the subgroup of $\operatorname{GL}(X)$ of elements $g$ such that $\left\langle v_{1} g, v_{2} g\right\rangle=\lambda_{g}\left\langle v_{1}, v_{2}\right\rangle$ for all $v_{1}, v_{2} \in X$, where $\lambda_{g} \in F^{*}$. The similitude factor $\lambda_{g}$ defines a homomorphism from $\operatorname{GSp}(X)$ to $F^{*}$ whose kernel is $\operatorname{Sp}(X)$. In Section 2B, we will extend $c(\cdot, \cdot)$ from $\operatorname{Sp}(X) \times \operatorname{Sp}(X)$ to $\mathrm{GSp}(X) \times \mathrm{GSp}(X)$. The result of this extension is that the set $\overline{\mathrm{GSp}(X)}=\mathrm{GSp}(X) \times\{ \pm 1\}$ is given a group structure by (2-1).

2A. Description of Rao's cocycle. We start with recalling some of the notations and facts that appear in Rao's formula. Detailed proofs can be found in [Rao 1993].

Let $E=\left\{e_{1}, e_{2}, \ldots, e_{n}, e_{1}^{*}, e_{2}^{*}, \ldots, e_{n}^{*}\right\}$ be a symplectic basis of $X$ : For $1 \leq i, j \leq$ $n$, we have $\left\langle e_{i}, e_{j}\right\rangle=\left\langle e_{i}^{*}, e_{j}^{*}\right\rangle=0$ and $\left\langle e_{i}, e_{j}^{*}\right\rangle=\delta_{i, j}$. In this basis, $\operatorname{Sp}(X)$ is realized as the set $\left\{A \in \operatorname{Mat}_{2 n \times 2 n}(F) \mid A J{ }^{\mathrm{t}} A=J\right\}$, where $J=\left(\left(0, I_{n}\right),\left(-I_{n}, 0\right)\right)$. Define $V=\operatorname{span}\left\{e_{1}, e_{2}, \ldots, e_{n}\right\}, V^{*}=\operatorname{span}\left\{e_{1}^{*}, e_{2}^{*}, \ldots, e_{n}^{*}\right\}$. These are two transverse Lagrangian subspaces. Let $P$ be the Siegel parabolic subgroup of $\operatorname{Sp}(X)$ consisting of the elements that preserve $V^{*}$. In coordinates,

$$
P=\left\{\left(\begin{array}{cc}
A & B \\
0 & { }^{\mathrm{t}} A^{-1}
\end{array}\right) \mid A \in \mathrm{GL}_{n}(F), B \in \operatorname{Mat}_{n \times n}(F),{ }^{\mathrm{t}} B=A^{-1} B{ }^{\mathrm{t}} A\right\} .
$$

Let $S$ be a subset of $\{1,2, \ldots, n\}$. We define $\tau_{S}, a_{S}$ to be the following elements of $\operatorname{Sp}(X)$ :

$$
\begin{aligned}
& e_{i} \cdot \tau_{S}=\left\{\begin{array}{rl}
-e_{i}^{*}, & i \in S, \\
e_{i}, & \text { otherwise, }
\end{array} \quad e_{i}^{*} \cdot \tau_{S}=\left\{\begin{aligned}
e_{i}, & i \in S, \\
e_{i}^{*}, & \text { otherwise },
\end{aligned}\right.\right. \\
& e_{i} \cdot a_{S}=\left\{\begin{array}{rl}
-e_{i}, & i \in S, \\
e_{i}, & \text { otherwise, }
\end{array} e_{i}^{*} \cdot a_{S}=\left\{\begin{aligned}
-e_{i}^{*}, & i \in S, \\
e_{i}^{*}, & \text { otherwise }
\end{aligned}\right.\right.
\end{aligned}
$$

The elements $\tau_{S}, a_{S}, \tau_{S^{\prime}}, a_{S^{\prime}}$ commute. Note that $a_{S} \in P, a_{S}^{2}=I_{2 n}$, and that $\tau_{S_{1}} \tau_{S_{2}}=\tau_{S_{1} \triangle S_{2}} a_{S_{1} \cap S_{2}}$, where $S_{1} \triangle S_{2}=S_{1} \cup S_{2} \backslash S_{1} \cap S_{2}$. In particular, $\tau_{S}^{2}=a_{S}$. For $S=\{1,2, \ldots, n\}$, we define $\tau=\tau_{S}$; in this case $a_{S}=-I_{2 n}$.

Define now $\Omega_{j}=\left\{\sigma \in \operatorname{Sp}(X) \mid \operatorname{dim}\left(V^{*} \cap V^{*} \sigma\right)=n-j\right\}$. Note that $P=\Omega_{0}$, $\tau_{S} \in \Omega_{|S|}$, and, more generally, if $\alpha, \beta, \gamma, \delta \in \operatorname{Mat}_{n \times n}(F)$ and $\sigma=((\alpha, \beta),(\gamma, \delta)) \in$ $\operatorname{Sp}(X)$, then $\sigma \in \Omega_{\operatorname{rank} \gamma}$. The Bruhat decomposition states that each $\Omega_{j}$ is a single double coset in ${ }_{P} \backslash \operatorname{Sp}(X) /{ }_{P}$, that $\Omega_{j}^{-1}=\Omega_{j}$, and that $\bigcup_{j=1}^{n} \Omega_{j}=\operatorname{Sp}(X)$. In particular, every element of $\operatorname{Sp}(X)$ has the form $p \tau_{S} p^{\prime}$, where $p, p^{\prime} \in P, S \subseteq$ $\{1,2, \ldots, n\}$. 
Let $p_{1}, p_{2} \in P$. Rao defines

$$
x\left(p_{1} \tau_{S} p_{2}\right) \equiv \operatorname{det}\left(\left.p_{1} p_{2}\right|_{V^{*}}\right) \quad\left(\bmod \left(F^{*}\right)^{2}\right)
$$

and proves that it is a well defined map from $\operatorname{Sp}(X)$ to $F^{*} /\left(F^{*}\right)^{2}$. Note that $x\left(a_{S}\right) \equiv$ $(-1)^{|S|}$, and, more generally, if $p=\left((A, B),\left(0,{ }^{\mathrm{t}} A^{-1}\right)\right) \in P$, then $x(p) \equiv \operatorname{det} A$. Also note that $x\left(\tau_{S}\right) \equiv 1$ and that for $g \in \Omega_{j}$, and $p_{1}, p_{2} \in P$,

$$
x\left(g^{-1}\right) \equiv x(g)(-1)^{j}, \quad x\left(p_{1} g p_{2}\right) \equiv x\left(p_{1}\right) x(g) x\left(p_{2}\right) .
$$

Theorem 5.3 in [Rao 1993] states that a nontrivial 2-cocycle on $\operatorname{Sp}(X)$ can be defined by

$$
\begin{aligned}
c\left(\sigma_{1}, \sigma_{2}\right)= & \left(x\left(\sigma_{1}\right), x\left(\sigma_{2}\right)\right)_{F}\left(-x\left(\sigma_{1}\right) x\left(\sigma_{2}\right), x\left(\sigma_{1} \sigma_{2}\right)\right)_{F} \\
& \cdot\left((-1)^{l}, d_{F}(\rho)\right)_{F}(-1,-1)_{F}^{l(l-1) / 2} h_{F}(\rho),
\end{aligned}
$$

where $\rho$ is the Leray invariant $-q\left(V^{*}, V^{*} \sigma_{1}, V^{*} \sigma_{2}^{-1}\right), d_{F}(\rho)$ and $h_{F}(\rho)$ are its discriminant and Hasse invariant, $(\cdot, \cdot)_{F}$ is the quadratic Hilbert symbol of $F$, and $2 l=j_{1}+j_{2}-j-\operatorname{dim} \rho$, where $\sigma_{1} \in \Omega_{j_{1}}, \sigma_{2} \in \Omega_{j_{2}}, \sigma_{1} \sigma_{2} \in \Omega_{j}$. We use Rao's normalization of the Hasse invariant. (Note that the cocycle formula just given differs slightly from the one appearing in Rao's paper, as there is a small mistake in its Theorem 5.3. A correction by Adams can be found in [Kudla 1994], Theorem 3.1). For future reference, we recall some of the properties of the Hilbert symbol:

$$
(a,-a)_{F}=1, \quad\left(a a^{\prime}, b\right)_{F}=(a, b)_{F}\left(a^{\prime}, b\right)_{F}, \quad(a, b)_{F}=(a,-a b)_{F} .
$$

From (2-2) and from previous remarks we obtain the following properties of $c(\cdot, \cdot)$. For $\sigma \in \Omega_{j}, p \in P$ we have

$$
\begin{aligned}
c\left(\sigma, \sigma^{-1}\right) & =\left(x(\sigma),(-1)^{j} x(\sigma)\right)_{F}(-1,-1)_{F}^{j(j-1) / 2}, \\
c(p, \sigma) & =c(\sigma, p)=(x(p), x(\sigma))_{F} .
\end{aligned}
$$

As a consequence of (2-5) and (2-3), we see that

$$
\left(p, \epsilon_{1}\right)(\sigma, \epsilon)\left(p, \epsilon_{1}\right)^{-1}=\left(p \sigma p^{-1}, \epsilon\right),
$$

for all $\sigma \in \operatorname{Sp}(X), p \in P$, and $\epsilon_{1}, \epsilon \in\{ \pm 1\}$.

Finally, we recall Corollary 5.6 in Rao's paper. For $S \subset\{1,2, \ldots, n\}$, define $X_{S}=\operatorname{span}\left\{e_{i}, e_{i}^{*} \mid i \in S\right\}$. We may now consider $x_{S}$ and $c_{X_{S}}(\cdot, \cdot)$ defined by analogy with $x$ and $c(\cdot, \cdot)$. Let $S_{1}$ and $S_{2}$ be a partition of $\{1,2, \ldots, n\}$. Suppose $\sigma_{1}, \sigma_{1}^{\prime} \in \operatorname{Sp}\left(X_{S_{1}}\right)$ and $\sigma_{2}, \sigma_{2}^{\prime} \in \operatorname{Sp}\left(X_{S_{2}}\right)$. Put $\sigma=\operatorname{diag}\left(\sigma_{1}, \sigma_{2}\right), \sigma^{\prime}=\operatorname{diag}\left(\sigma_{1}^{\prime}, \sigma_{2}^{\prime}\right)$. Rao proves that

$$
\begin{aligned}
& c\left(\sigma, \sigma^{\prime}\right)=c_{S_{1}}\left(\sigma_{1}, \sigma_{1}^{\prime}\right) c_{S_{2}}\left(\sigma_{2}, \sigma_{2}^{\prime}\right)\left(x_{S_{1}}\left(\sigma_{1}\right), x_{S_{2}}\left(\sigma_{2}\right)\right)_{F} \\
& \cdot\left(x_{S_{1}}\left(\sigma_{1}^{\prime}\right), x_{S_{2}}\left(\sigma_{2}^{\prime}\right)\right)_{F}\left(x_{S_{1}}\left(\sigma_{1} \sigma_{1}^{\prime}\right), x_{S_{2}}\left(\sigma_{2} \sigma_{2}^{\prime}\right)\right)_{F} .
\end{aligned}
$$


2B. Extension of Rao's cocycle to $\mathbf{G S p}(X) . \quad F^{*}$ is embedded in $\operatorname{GSp}(X)$ via

$$
\lambda \mapsto i(\lambda)=\left(\begin{array}{cc}
I_{n} & 0 \\
0 & \lambda I_{n}
\end{array}\right) .
$$

Using this embedding we define an action of $F^{*}$ on $\operatorname{Sp}(X):(g, \lambda) \mapsto g^{\lambda}=$ $i\left(\lambda^{-1}\right) g i(\lambda)$. Let $F^{*} \ltimes \operatorname{Sp}(X)$ be the semidirect product corresponding to this action. For $\alpha, \beta, \gamma, \delta \in \operatorname{Mat}_{n \times n}(F)$ and $g=\left(\begin{array}{ll}\alpha & \beta \\ \gamma & \delta\end{array}\right) \in \operatorname{GSp}(X)$, define

$$
p(g)=\left(\begin{array}{cc}
\alpha & \beta \\
\lambda_{g}^{-1} \gamma & \lambda_{g}^{-1} \delta
\end{array}\right) \in \operatorname{Sp}(X),
$$

and note that $g=i\left(\lambda_{g}\right) p(g)$. The map $g \mapsto \iota(g)=\left(\lambda_{g}, p(g)\right)$ is an isomorphism between $\operatorname{GSp}(X)$ and $F^{*} \ltimes \operatorname{Sp}(X)$.

From the theory of the Weil representation (see [Mœglin et al. 1987, page 36]), we know that for any $\lambda \in F^{*}$, we can define a map $v_{\lambda}: \operatorname{Sp}(X) \rightarrow\{ \pm 1\}$ such that $(g, \epsilon) \mapsto(g, \epsilon)^{\lambda}=\left(g^{\lambda}, \epsilon v_{\lambda}(g)\right)$ is an automorphism of $\overline{\operatorname{Sp}(X)}$. In $2 \mathrm{~B}$, we compute $v_{\lambda}$. We shall also show there that $(\lambda,(g, \epsilon)) \mapsto(g, \epsilon)^{\lambda}$ defines an action of $F^{*}$ on $\overline{\operatorname{Sp}(X)}$. Here we just want to show how this computation enables us to extend $c(\cdot, \cdot)$ to a 2-cocycle $\tilde{c}(\cdot, \cdot)$ on $\operatorname{GSp}(X)$ and hence write an explicit multiplication formula of $\overline{\operatorname{GSp}(X)}$, the metaplectic double cover of $\operatorname{GSp}(X)$. We define the group $F^{*} \ltimes \overline{\mathrm{Sp}(X)}$ using the multiplication

$$
\left(a,\left(g, \epsilon_{1}\right)\right)\left(b,\left(h, \epsilon_{2}\right)\right)=\left(a b,\left(g, \epsilon_{1}\right)^{b}\left(h, \epsilon_{2}\right)\right) .
$$

We also define a bijection from $\operatorname{GSp}(X) \times\{ \pm 1\}$ to $F^{*} \times \overline{\operatorname{Sp}(X)}$ by $\bar{l}(g, \epsilon)=$ $\left(\lambda_{g},(p(g), \epsilon)\right)$, whose inverse is given by $\bar{\imath}^{-1}(\lambda,(h, \epsilon))=(i(\lambda) h, \epsilon)$. We use $\bar{\imath}$ to define a group structure on $\operatorname{GSp}(X) \times\{ \pm 1\}$. A straightforward computation will show that the multiplication in $\overline{\mathrm{GSp}(X)}$ is given by

$$
\left(g, \epsilon_{1}\right)\left(h, \epsilon_{2}\right)=\left(g h, v_{\lambda_{h}}(p(g)) c\left(p(g)^{\lambda_{h}}, p(h) \epsilon_{1} \epsilon_{2}\right)\right) .
$$

Thus,

$$
\tilde{c}(g, h)=v_{\lambda_{h}}(p(g)) c\left(p(g)^{\lambda_{h}}, p(h)\right)
$$

serves as a nontrivial 2-cocycle on $\operatorname{GSp}(X)$. We remark here that Kubota [1969] (see also [Gelbart 1976]) used a similar construction to extend a nontrivial double cover of $\mathrm{SL}_{2}(F)$ to a nontrivial double cover of $\mathrm{GL}_{2}(F)$. For $n=1$, our construction agrees with Kubota's.

Computation of $v_{\lambda}(g)$. Barthel [1991] extended Rao's unnormalized cocycle to $\operatorname{GSp}(X)$. One may compute $v_{\lambda}(g)$ using Barthel's work and Rao's normalizing factors. Instead, we compute $v_{\lambda}(g)$ using Rao's (normalized) cocycle. Fix $\lambda \in F^{*}$. 
Since $(g, \epsilon) \mapsto(g, \epsilon)^{\lambda}$ is an automorphism, $v_{\lambda}$ satisfies

$$
v_{\lambda}(g) v_{\lambda}(h) v_{\lambda}(g h)=\frac{c\left(g^{\lambda}, h^{\lambda}\right)}{c(g, h)} .
$$

We shall show that this property determines $v_{\lambda}$.

We first note that

$$
\left(\begin{array}{cc}
A & B \\
0 & { }^{\mathrm{t}} A^{-1}
\end{array}\right)^{\lambda}=\left(\begin{array}{cc}
A & \lambda B \\
0 & { }^{\mathrm{t}} A^{-1}
\end{array}\right), \quad x\left(\left(\begin{array}{cc}
A & B \\
0 & { }^{\mathrm{t}} A^{-1}
\end{array}\right)^{\lambda}\right) \equiv x\left(\left(\begin{array}{cc}
A & B \\
0 & { }^{\mathrm{t}} A^{-1}
\end{array}\right)\right) .
$$

For $S \subseteq\{1,2, \ldots, n\}$ define $a_{S}(\lambda) \in P$ by

$$
e_{i} \cdot a_{S}(\lambda)=\left\{\begin{array}{rl}
\lambda^{-1} e_{i}, & i \in S, \\
e_{i}, & \text { otherwise, }
\end{array} \quad e_{i}^{*} \cdot a_{S}(\lambda)=\left\{\begin{aligned}
\lambda e_{i}^{*}, & i \in S, \\
e_{i}^{*}, & \text { otherwise } .
\end{aligned}\right.\right.
$$

Note that $a_{S}=a_{S}(-1)$. One can verify that

$$
\tau_{S}^{\lambda}=a_{S}(\lambda) \tau_{S}=\tau_{S} a_{S}\left(\lambda^{-1}\right)
$$

Since $x\left(a_{S}(\lambda)\right) \equiv \lambda^{|S|}$, we obtain, using (2-9) and (2-10), the Bruhat decomposition, and the properties of $x$ presented earlier, that $\Omega_{j}^{\lambda}=\Omega_{j}$ and that, for $g \in \Omega_{j}$,

$$
x\left(g^{\lambda}\right) \equiv \lambda^{j} x(g) .
$$

Lemma 2.1. For $p \in P, g \in \Omega_{j}$ we have

$$
v_{\lambda}(p) v_{\lambda}(g) v_{\lambda}(p g)=\left(x(p), \lambda^{j}\right)_{F}
$$

and

$$
v_{\lambda}(g) v_{\lambda}(p) v_{\lambda}(g p)=\left(x(p), \lambda^{j}\right)_{F} .
$$

Proof. We prove (2-12) only. (2-13) follows in the same way. We use (2-8), (2-5), (2-11) and (2-3):

$$
\begin{aligned}
v_{\lambda}(p) v_{\lambda}(g) v_{\lambda}(p g) & =\frac{c\left(p^{\lambda}, g^{\lambda}\right)}{c(p, g)}=\frac{\left(x\left(p^{\lambda}\right), x\left(g^{\lambda}\right)\right)_{F}}{(x(p), x(g))_{F}} \\
& =\frac{\left(x(p), x(g) \lambda^{j}\right)_{F}}{(x(p), x(g))_{F}}=\left(x(p), \lambda^{j}\right)_{F} .
\end{aligned}
$$

Lemma 2.2. There exists a unique $t_{\lambda} \in F^{*} / F^{* 2}$ such that $v_{\lambda}(p)=\left(x(p), t_{\lambda}\right)_{F}$ for all $p \in P$. 
Proof. Substituting $p^{\prime} \in P$ for $g$ in (2-12) we see that $\left.v_{\lambda}\right|_{P}$ is a quadratic character. Since $N$, the unipotent radical of $P$, is isomorphic to a vector space over $F$, it follows that $N^{2}=N$. Thus, $\left.v_{\lambda}\right|_{N}$ is trivial. We conclude that $\left.v_{\lambda}\right|_{P}$ is a quadratic character of $\mathrm{GL}_{n}(F)$ extended to $P$. Every quadratic character of $\mathrm{GL}_{n}(F)$ is of the form $g \mapsto \chi(\operatorname{det} g)$, where $\chi$ is a quadratic character of $F^{*}$. The nondegeneracy of the Hilbert symbol implies every such character of $F^{*}$ has the form $\chi(a)=\left(a, t_{\chi}\right)_{F}$, where $t_{\chi} \in F^{*}$ uniquely determined by $\chi$ up to multiplication by squares.

Lemma 2.3. For $\sigma \in \Omega_{j}$ we have $v_{\lambda}(\sigma)=\left(x(\sigma), t_{\lambda} \lambda^{j}\right)_{F} v_{\lambda}\left(\tau_{S}\right)$, where $S \subseteq$ $\{1,2, \ldots, n\}$ is such that $|S|=j$. In particular, if $|S|=\left|S^{\prime}\right|$ then $v_{\lambda}\left(\tau_{S}\right)=v_{\lambda}\left(\tau_{S^{\prime}}\right)$.

Proof. An element $\sigma \in \Omega_{j}$ has the form $\sigma=p \tau_{S} p^{\prime}$, where $p, p^{\prime} \in P$ and $|S|=j$. Substituting $g=\tau_{S} p^{\prime}$ in (2-12) yields $v_{\lambda}\left(p \tau_{S} p^{\prime}\right)=v_{\lambda}(p) v_{\lambda}\left(\tau_{S} p^{\prime}\right)\left(x(p), \lambda^{j}\right)_{F}$, while substituting $g=\tau_{S}$ and $p=p^{\prime}$ in (2-13) yields $v_{\lambda}\left(\tau_{S} p^{\prime}\right)=v_{\lambda}\left(\tau_{S}\right) v_{\lambda}\left(p^{\prime}\right)$. $\left(x\left(p^{\prime}\right), \lambda^{j}\right)_{F}$. Using these two equalities together with (2-3) and Lemma 2.2, we obtain $v_{\lambda}\left(p \tau_{S} p^{\prime}\right)=\left(x\left(p p^{\prime}\right), t_{\lambda} \lambda^{j}\right)_{F} v_{\lambda}\left(\tau_{S}\right)$. Since $|S|=\left|S^{\prime}\right|$ implies $p \tau_{S} p^{-1}=\tau_{s^{\prime}}$, for some $p \in P$, the last argument shows that $v_{\lambda}\left(\tau_{S}\right)=v_{\lambda}\left(\tau_{S^{\prime}}\right)$.

It is clear now that, once we compute $t_{\lambda}$ and $v_{\lambda}\left(\tau_{S}\right)$ for all $S \subseteq\{1,2, \ldots, n\}$, we will find the explicit formula for $v_{\lambda}$.

Lemma 2.4. $t_{\lambda}=\lambda$ and $v_{\lambda}\left(\tau_{S}\right)=(\lambda, \lambda)_{F}^{|S|(|S|-1) / 2}$.

Proof. Let $k$ be a symmetric matrix in $\mathrm{GL}_{n}(F)$. Put

$$
p_{k}=\left(\begin{array}{cc}
k & -I_{n} \\
0 & k^{-1}
\end{array}\right) \in P, \quad n_{k}=\left(\begin{array}{cc}
I_{n} & k \\
0 & I_{n}
\end{array}\right) \in N,
$$

and note that $x\left(n_{k}\right) \equiv 1, x\left(p_{k}\right) \equiv \operatorname{det} k$, and that

$$
\tau n_{k} \tau=n_{-k^{-1}} \tau p_{k} .
$$

We are going to compute $v_{\lambda}(\tau) v_{\lambda}\left(n_{k} \tau\right) v_{\lambda}\left(\tau n_{k} \tau\right)$ in two ways: First, by Lemma 2.3 and by (2-14), we have $v_{\lambda}(\tau) v_{\lambda}\left(n_{k} \tau\right) v_{\lambda}\left(\tau n_{k} \tau\right)=v_{\lambda}(\tau) v_{\lambda}(\tau) v_{\lambda}\left(n_{-k^{-1}} \tau p_{k}\right)=$ $v_{\lambda}\left(n_{-k^{-1}} \tau p_{k}\right)$. Since

$$
x\left(\tau n_{k} \tau\right) \equiv x\left(n_{-k^{-1}} \tau p_{k}\right) \equiv \operatorname{det}(k),
$$

we obtain, using Lemma 2.3 again,

$$
v_{\lambda}(\tau) v_{\lambda}\left(n_{k} \tau\right) v_{\lambda}\left(\tau n_{k} \tau\right)=\left(\operatorname{det} k, t_{\lambda} \lambda^{n}\right)_{F} v_{\lambda}(\tau) .
$$

Second, by (2-8) we have

$$
v_{\lambda}(\tau) v_{\lambda}\left(n_{k} \tau\right) v_{\lambda}\left(\tau n_{k} \tau\right)=\frac{c\left(\tau, n_{k} \tau\right)}{c\left(\tau^{\lambda},\left(n_{k} \tau\right)^{\lambda}\right)} .
$$


We shall compute the two terms on the right side of (2-17), starting with $c\left(\tau, n_{k} \tau\right)$. Let $\rho$ and $l$ be the factors in (2-2), where $\sigma_{1}=\tau, \sigma_{2}=n_{k} \tau$. Recall that the Leray invariant is stable under the action of $\operatorname{Sp}(X)$ on Lagrangian triplets, see [Rao 1993, Theorem 2.11]. Hence,

$$
q\left(V^{*}, V^{*} \tau, V^{*}\left(n_{k} \tau\right)^{-1}\right)=q\left(V^{*}, V, V^{*}\left(-I_{2 n} \tau n_{-k}\right)\right)=q\left(V^{*}, V, V n_{-k}\right) .
$$

We conclude that $\rho=k, l=0$. Using (2-2), and (2-15) we observe that

$$
c\left(\tau, n_{k} \tau\right)=(-1, \operatorname{det} k)_{F} h_{F}(k) .
$$

We now turn to $c\left(\tau^{\lambda},\left(n_{k} \tau\right)^{\lambda}\right)$. Let $\rho$ and $l$ be the factors in (2-2), where $\sigma_{1}=\tau^{\lambda}$ and $\sigma_{2}=\left(n_{k} \tau\right)^{\lambda}$. Note that (2-9) and (2-10) imply $\left(n_{k} \tau\right)^{\lambda}=n_{\lambda k} \lambda I_{2 n} \tau$, and hence

$$
q\left(\left(V^{*}, V^{*} \tau^{\lambda}, V^{*}\left(\left(n_{k} \tau\right)^{\lambda}\right)^{-1}\right)=q\left(V^{*}, V, V n_{-\lambda k}\right) .\right.
$$

Therefore $\rho=m k$ and $l=0$, and so we get

$$
c\left(\tau^{\lambda},\left(n_{k} \tau\right)^{\lambda}\right)=\left(x\left(\lambda I_{2 n}\right), x\left(\lambda I_{2 n}\right)\right)_{F}\left(-1, x\left(\tau^{\lambda}\left(n_{k} \tau\right)^{\lambda}\right)\right)_{F} h_{F}(\lambda k) .
$$

We recall (2-14) and note now that

$$
\tau^{\lambda}\left(n_{k} \tau\right)^{\lambda}=\left(\tau n_{k} \tau\right)^{\lambda}=\left(n_{-k^{-1}} \tau p_{k}\right)^{\lambda}=n_{-\lambda k^{-1}} \lambda I_{2 n} \tau\left(\begin{array}{cc}
k & \lambda k \\
0 & k^{-1}
\end{array}\right) .
$$

Hence, $c\left(\tau^{\lambda},\left(n_{k} \tau\right)^{\lambda}\right)=\left(\lambda^{n}, \lambda^{n}\right)_{F}\left(-1, \lambda^{n} \operatorname{det} k\right)_{F} h_{F}(\lambda k)$, or, using (2-3):

$$
c\left(\tau^{\lambda},\left(n_{k} \tau\right)^{\lambda}\right)=(-1, \operatorname{det} k)_{F} h_{F}(\lambda k) .
$$

Using (2-16), (2-17), (2-18) and (2-19) we finally get

$$
v_{\lambda}(\tau)\left(\operatorname{det} k, t_{\lambda} \lambda^{n}\right)_{F}=\frac{h_{F}(\lambda k)}{h_{F}(k)} .
$$

By substituting $k=I_{n}$ in the above, we get $v_{\lambda}(\tau)=(\lambda, \lambda)_{F}^{n(n-1) / 2}$, and we can rewrite it as

$$
\left(\operatorname{det} k, t_{\lambda} \lambda^{n}\right)_{F}=\frac{h_{F}(\lambda k)}{h_{F}(k)}(\lambda, \lambda)_{F}^{n(n-1) / 2} .
$$

To find $t_{\lambda}$, we note that for any $y \in F^{*}$ we can put $k_{y}=\operatorname{diag}(1,1, \ldots, y)$ in $(2-20)$ and obtain $\left(y, t_{\lambda} \lambda^{n}\right)_{F}=(\lambda, \lambda)_{F}^{(n-1)(n-2) / 2}(\lambda, \lambda y)_{F}^{n-1}(\lambda, \lambda)_{F}^{n(n-1) / 2}$. For both even and odd $n$, this is equivalent to $(y, \lambda)_{F}=\left(y, t_{\lambda}\right)_{F}$. That the last equality holds for all $y \in F^{*}$ implies that $t_{\lambda} \equiv \lambda\left(\bmod \left(F^{*}\right)^{2}\right)$.

We are left to compute $v_{\lambda}\left(\tau_{S}\right)$ for $S \subsetneq\{1,2, \ldots, n\}$. For such $S$, define ${ }_{S} \tau \in$ $\operatorname{Sp}\left(X_{S}\right)$ by analogy with $\tau \in \mathrm{Sp}(X)$. We can embed $\operatorname{Sp}\left(X_{S}\right)$ in $\operatorname{Sp}(X)$ in a way that maps ${ }_{S} \tau$ to $\tau_{S}$. We may now use (2-7) and repeat the computation of $v_{\lambda}(\tau)$. 
Joining Lemma 2.3, and Lemma 2.4, we write the explicit formula for $v_{\lambda}$. For $g \in \Omega_{j}$ we have

$$
v_{\lambda}(g)=\left(x(g), \lambda^{j+1}\right)_{F}(\lambda, \lambda)^{j(j-1) / 2} .
$$

One can easily check now that $v_{\lambda}(g) v_{\eta}\left(g^{\lambda}\right)=v_{\lambda \eta}(g)$ and conclude that the map $(\lambda,(g, \epsilon)) \mapsto(g, \epsilon)^{\lambda}$ defines an action of $F^{*}$ on $\overline{\operatorname{Sp}(X)}$, namely $\left((g, \epsilon)^{\lambda}\right)^{\eta}=$ $(g, \epsilon)^{\lambda \eta}$. Lastly, comparing (2-21) and (2-4), keeping (2-3) in mind, we note that

$$
v_{-1}(g)=v_{-1}\left(g^{-1}\right)=c\left(g, g^{-1}\right) .
$$

This fact will play an important role in the proof of the uniqueness of Whittaker models for $\overline{\mathrm{Sp}(X)}$.

\section{Uniqueness of the Whittaker model}

3A. Statement of the main results. Let $\psi$ be a nontrivial character of $F$. Let $Z^{\prime}$ be the group of upper triangular unipotent matrices in $\mathrm{GL}_{n}(F)$. Let $Z$ be the subgroup of $\operatorname{Sp}_{2 n}(F)$ that consists of elements of the form $\left((z, b),\left(0,{ }^{\mathrm{t}} z^{-1}\right)\right)$ in which $z \in Z^{\prime}$ and $b \in \operatorname{Mat}_{n \times n}(F)$ satisfy $b=z^{-1} b{ }^{\mathrm{t}} z$. We shall continue to denote by $\psi$ the character of $Z$ given by $\psi(z)=\psi\left(b_{(n, n)}+\sum_{i=1}^{n-1} z_{(i, i+1)}\right)$. Let $\bar{Z}$ be the inverse image of $Z$ in $\overline{\operatorname{Sp}_{2 n}(F)}$. From (2-5) it follows that $\bar{Z} \simeq Z \times\{ \pm 1\}$. We define a character of $\bar{Z}$ by $(z, \epsilon) \mapsto \epsilon \psi(z)$ and continue to denote it by $\psi$.

Let $\left(\pi, V_{\pi}\right)$ be a smooth representation of $\overline{\mathrm{Sp}_{2 n}(F)}$. By a Whittaker functional on $\pi$ with respect to $\psi$, we mean a linear functional $w$ on $V_{\pi}$ satisfying $w(\pi(z) v)=\psi(z) w(v)$ for all $v \in V_{\pi}, z \in \bar{Z}$. Define $W_{\pi, \psi}$ to be the space of Whittaker functionals on $\pi$ with respect to $\psi$. We define $\hat{\pi}$ as the (smooth) dual representation to $\pi$.

Theorem 3.1. If $\left(\pi, V_{\pi}\right)$ is an irreducible admissible representation of $\overline{\operatorname{Sp}_{2 n}(F)}$, then

$$
\operatorname{dim}\left(W_{\pi, \psi}\right) \cdot \operatorname{dim}\left(W_{\hat{\pi}, \psi^{-1}}\right) \leq 1 .
$$

The proof of this theorem will show:

Theorem 3.2. Suppose $\left(\pi, V_{\pi}\right)$ is an irreducible admissible representation of

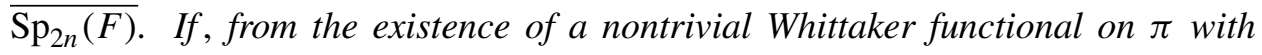
respect to $\psi$, one can deduce the existence of a nontrivial Whittaker functional on $\hat{\pi}$ with respect to $\psi^{-1}$, then $\operatorname{dim}\left(W_{\pi, \psi}\right) \leq 1$.

Corollary 3.3. If $\left(\pi, V_{\pi}\right)$ is an irreducible admissible unitary representation of $\overline{\operatorname{Sp}_{2 n}(F)}$, then $\operatorname{dim}\left(W_{\pi, \psi}\right) \leq 1$.

Proof of the corollary. We show that the conditions of Theorem 3.2 hold in this case. Indeed, if $\left(\pi, V_{\pi}\right)$ is an irreducible admissible unitary representation of $\overline{\mathrm{Sp}_{2 n}(F)}$, one can realize the dual representation in the space $\widetilde{V_{\pi}}$, which is identical 
to $V_{\pi}$ as a commutative group. The scalars act on $\widetilde{V_{\pi}}$ by $\lambda \cdot v=\bar{\lambda} v$. The action of $\hat{\pi}$ in this realization is given by $\hat{\pi}(g)=\pi(g)$. It is clear now that if $L$ is a nontrivial Whittaker functional on $\pi$ with respect to $\psi$ then $L$, acting on $\widetilde{V_{\pi}}$, is a nontrivial Whittaker functional on $\hat{\pi}$ with respect to $\psi^{-1}$.

Since every cuspidal representation $\pi$ is unitary, it follows from Corollary 3.3 that $\operatorname{dim}\left(W_{\pi, \psi}\right) \leq 1$. Furthermore, assume now that $\pi$ is an irreducible admissible representation of $\overline{\operatorname{Sp}_{2 n}(F)}$. Then $\pi$ is a subquotient of a representation induced from a cuspidal representation of a parabolic subgroup. Let $H$ be a parabolic subgroup of $\operatorname{Sp}_{2 n}(F)$. It is known [Mœglin et al. 1987, page 39] that if $g$ and $h$ commute in $\operatorname{Sp}_{2 n}(F)$ then $\left(g, \epsilon_{1}\right)$ and $\left(h, \epsilon_{2}\right)$ commute in $\overline{\operatorname{Sp}_{2 n}(F)}$. Therefore, $\bar{M}_{H}$, the inverse image of the Levy part of $H$, is isomorphic to

$$
\mathrm{GL}_{n_{1}}(F) \times \mathrm{GL}_{n_{2}}(F) \times \cdots \times \mathrm{GL}_{n_{r}}(F) \times \overline{\mathrm{Sp}_{2 k}(F)} .
$$

Suppose that for $1 \leq i \leq r$, the representation $\sigma_{i}$ of $\mathrm{GL}_{n_{i}}(F)$ is cuspidal, and that $\pi^{\prime}$ is a cuspidal representation of $\overline{\operatorname{Sp}_{2 k}(F)}$. Denote by $\psi_{i}$ and $\psi^{\prime}$ the restrictions of $\psi$ to the unipotent radicals of $\mathrm{GL}_{n_{i}}$ and $\overline{\mathrm{Sp}_{2 k}(F)}$, respectively, embedded in $\bar{M}_{H}$. Note that $\operatorname{dim}\left(W_{\sigma_{i}, \psi_{i}}\right) \leq 1$ and $\operatorname{dim}\left(W_{\pi^{\prime}, \psi^{\prime}}\right) \leq 1$. Let $\tau$ be the representation of $\bar{M}_{H}$ defined by

$$
\begin{aligned}
&\left(\operatorname{diag}\left(g_{1}, g_{2}, \ldots, g_{r}, h,{ }^{\mathrm{t}} g_{r}^{-1},{ }^{\mathrm{t}} g_{r-1}^{-1}, \ldots,{ }^{\mathrm{t}} g_{1}^{-1}\right), \epsilon\right) \mapsto \\
& \bigotimes_{i=1}^{i=r} \sigma_{i}\left(g_{i}\right) \gamma_{\psi}\left(\operatorname{det} g_{i}\right) \otimes \pi(h, \epsilon),
\end{aligned}
$$

where $\gamma_{\psi}$ is the Weil index of $\psi$ (for details on $\gamma_{\psi}$ see [Rao 1993, appendix]). We extend $\tau$ from $M_{H}$ to $\bar{H}$, letting the unipotent radical act trivially. Define $\operatorname{Ind}\left(\bar{H}, \overline{\operatorname{Sp}_{2 n}(F)}, \tau\right)$ to be the corresponding induced representation. One may use the methods of [Rodier 1973], extended in [Banks 1998] to a nonalgebraic setting, and conclude that

$$
\operatorname{dim}\left(W_{\operatorname{Ind}\left(\bar{H}, \overline{\operatorname{Sp}_{2 n}(F)}, \tau\right), \psi}\right)=\operatorname{dim}\left(W_{\pi^{\prime}, \psi^{\prime}}\right) \Pi_{i=1}^{i=r} \operatorname{dim}\left(W_{\sigma_{i}, \psi_{i}}\right) .
$$

Now, if $V_{2} \subseteq V_{1} \subseteq \operatorname{Ind}\left(\bar{H}, \overline{\mathrm{Sp}_{2 n}(F)}, \tau\right)$, are two $\overline{\mathrm{Sp}_{2 n}(F)}$ modules then clearly the dimension of the Whittaker functionals on $V_{1}$ and $V_{2}$ with respect to $\psi$ is not greater then $\operatorname{dim}\left(W_{\operatorname{Ind}\left(\bar{H}, \overline{\mathrm{Sp}_{2 n}(F)}, \tau\right), \psi}\right)$. It follows now that $\operatorname{dim}\left(W_{\pi, \psi}\right) \leq 1$. Thus, we proved

Theorem 3.4. Let $\pi$ be an irreducible, admissible representation of $\overline{\operatorname{Sp}_{2 n}(F)}$. Then $\operatorname{dim}\left(W_{\pi, \psi}\right) \leq 1$.

Proof of Theorem 3.1. Define on $\operatorname{Sp}_{2 n}(F)$ the map

$$
g \mapsto{ }^{\tau} g=\sigma_{0}\left({ }^{\mathrm{t}} g\right) \sigma_{0}^{-1},
$$


where

$$
\sigma_{0}=\left(\begin{array}{cc}
0 & \epsilon_{n} \\
\epsilon_{n} & 0
\end{array}\right), \quad \epsilon_{n}=\operatorname{diag}\left(1,-1,1 \ldots,(-1)^{n+1}\right) \in \mathrm{GL}_{n}(F)
$$

We note that $\sigma_{0}^{-1}={ }^{\mathrm{t}} \sigma_{0}=\sigma_{0}$, and that $\sigma_{0} \in \mathrm{GSp}_{2 n}(F)$ with similitude factor -1 . Hence, $g \mapsto{ }^{\tau} g$ is an antiautomorphism of $\operatorname{Sp}_{2 n}(F)$ of order 2 of. We now extend $\tau$ to $\overline{\mathrm{Sp}_{2 n}(F)}$. A similar lifting was used in [Gelbart et al. 1979] for $\overline{\mathrm{GL}_{2}(F)}$.

Lemma 3.5. The map $(g, \epsilon) \mapsto{ }^{\bar{\tau}}(g, \epsilon)=\left({ }^{\tau} g, \epsilon\right)$ is an antiautomorphism of $\overline{\mathrm{Sp}_{2 n}(F)}$ of order 2 . It preserves both $\bar{Z}$ and $\psi$ and satisfies ${ }^{\bar{\tau}}\left(\operatorname{Sp}_{2 n}(F), \epsilon\right)=$ $\left(\mathrm{Sp}_{2 n}(F), \epsilon\right)$.

Proof. We note that if $g \in \mathrm{Sp}_{2 n}(F)$ then ${ }^{\mathrm{t}} g=-J g^{-1} J$. Hence,

$$
{ }^{\tau} g=\left(\begin{array}{cc}
\epsilon_{n} & 0 \\
0 & \epsilon_{n}
\end{array}\right)\left(\begin{array}{cc}
-I_{n} & 0 \\
0 & I_{n}
\end{array}\right) g^{-1}\left(\begin{array}{cc}
-I_{n} & 0 \\
0 & I_{n}
\end{array}\right)\left(\begin{array}{cc}
\epsilon_{n} & 0 \\
0 & \epsilon_{n}
\end{array}\right)^{-1} .
$$

Thus, the map

$$
(g, \epsilon) \mapsto\left({ }^{\tau} g, \epsilon c\left(g, g^{-1}\right) v_{-1}\left(g^{-1}\right) c\left(p_{\epsilon}, \tilde{g}\right) c\left(p_{\epsilon} \tilde{g}, p_{\epsilon}^{-1}\right) c\left(p_{\epsilon}, p_{\epsilon}^{-1}\right)\right),
$$

where

$$
p_{\epsilon}=\left(\begin{array}{cc}
\epsilon_{n} & 0 \\
0 & \epsilon_{n}
\end{array}\right) \in P \quad \text { and } \quad \tilde{g}=\left(\begin{array}{cc}
-I_{n} & 0 \\
0 & I_{n}
\end{array}\right) g^{-1}\left(\begin{array}{cc}
-I_{n} & 0 \\
0 & I_{n}
\end{array}\right)
$$

is an antiautomorphism of $\overline{\mathrm{Sp}_{2 n}(F)}$. We now show that

$$
c\left(g, g^{-1}\right) v_{-1}\left(g^{-1}\right) c\left(p_{\epsilon}, \tilde{g}\right) c\left(p_{\epsilon} \tilde{g}, p_{\epsilon}^{-1}\right) c\left(p_{\epsilon}, p_{\epsilon}^{-1}\right)=1 .
$$

Indeed, $c\left(p_{\epsilon}, \tilde{g}\right) c\left(p_{\epsilon} \tilde{g}, p_{\epsilon}^{-1}\right) c\left(p_{\epsilon}, p_{\epsilon}^{-1}\right)=1$ is a property of Rao's cocycle noted in (2-6). The fact that $c\left(g, g^{-1}\right) v_{-1}\left(g^{-1}\right)=1$ is a consequence of the calculation of $v_{\lambda}(g)$ and is noted in (2-22). The remaining assertions of this lemma are clear.

Let $S\left(\overline{\operatorname{Sp}_{2 n}(F)}\right)$ be the space of Schwartz functions on $\overline{\mathrm{Sp}_{2 n}(F)}$. For $h \in \overline{\operatorname{Sp}_{2 n}(F)}$ and $\phi \in S\left(\overline{\operatorname{Sp}_{2 n}(F)}\right)$, we define $\lambda(h) \phi, \rho(h) \phi$, and ${ }^{\bar{\tau}} \phi$ by

$$
(\rho(h) \phi)(g)=\phi(g h), \quad(\lambda(h) \phi)(g)=\phi\left(h^{-1} g\right), \quad{ }^{\bar{\tau}} \phi(g)=\phi\left({ }^{\bar{\tau}} g\right) .
$$

These maps are elements of $S\left(\overline{\operatorname{Sp}_{2 n}(F)}\right)$. We give $S\left(\overline{\operatorname{Sp}_{2 n}(F)}\right)$ an algebra structure, called the Hecke algebra, by the convolution. Given $\left(\pi, V_{\pi}\right)$, a representation of $\overline{\operatorname{Sp}_{2 n}(F)}$, we define as usual a representation of this algebra in the space $V_{\pi}$ by

$$
\pi(\phi) v=\int_{\overline{S p}_{2 n}(F)} \phi(g) \pi(g) v d g .
$$

The following theorem, known as the Gelfand-Kazhdan Theorem in the context of $\mathrm{GL}_{n}(F)$ (see [Gel'fand and Kazhdan 1975; Bernstein and Zelevinskii 1976]), will be used in the proof of Theorem 3.1. 
Theorem 3.6. Suppose $D$ is a functional on $S\left(\overline{\operatorname{Sp}_{2 n}(F)}\right)$ with $D\left(\lambda\left(z_{1}\right) \rho\left(z_{2}\right) \phi\right)=$ $\psi\left(z_{2} z_{1}^{-1}\right) D(\phi)$ for all $\phi \in S\left(\overline{\mathrm{Sp}_{2 n}(F)}\right)$ and $z_{1}, z_{1} \in \bar{Z}$. Then $D$ is $\bar{\tau}$ invariant, that is, $D\left({ }^{\bar{\tau}} \phi\right)=D(\phi)$ for all $\phi \in S\left(\overline{\operatorname{Sp}_{2 n}(F)}\right)$.

We will prove this theorem in Section 3B. Here we use it for:

Proof of Theorem 3.1. Since any irreducible admissible representation of $\overline{\operatorname{Sp}_{2 n}(F)}$ may be realized as a dual representation, the proof amounts to showing that if $W_{\pi, \psi} \neq 0$ then $\operatorname{dim} W_{\hat{\pi}, \psi^{-1}} \leq 1$. We shall use an argument similar to the one in [Soudry 1987, Theorem 2.1]. Let $w$ be a nontrivial Whittaker functional on $\left(\pi, V_{\pi}\right)$ with respect to $\psi$. Suppose $\widehat{w}_{1}$ and $\widehat{w}_{2}$ are two nontrivial Whittaker functionals on $\hat{\pi}$ with respect to $\psi^{-1}$. The proof will be achieved once we show that $\widehat{w}_{1}$ and $\widehat{w}_{2}$ are proportional.

For $\phi \in S\left(\overline{\operatorname{Sp}_{2 n}(F)}\right)$, let $\pi^{*}(\phi) w$ be a functional on $V_{\pi}$ defined by

$$
\left(\pi^{*}(\phi) w\right) v=\int_{\overline{\mathrm{Sp}_{2 n}(F)}} \phi(g) w\left(\pi\left(g^{-1}\right) v\right) d g .
$$

Note that $\phi \in S\left(\overline{\operatorname{Sp}_{2 n}(F)}\right)$ implies $\pi^{*}(\phi) w$ is smooth even if $w$ is not. Thus, $\pi^{*}(\phi) w$ lies in $\in V_{\hat{\pi}}$. By a change of variables we have

$$
\hat{\pi}(h)\left(\pi^{*}(\phi) w\right)=\pi^{*}(\lambda(h) \phi) w .
$$

Now define $R_{1}$ and $R_{2}$, two functionals on $S\left(\overline{\operatorname{Sp}_{2 n}(F)}\right)$, through

$$
R_{i}(\phi)=\widehat{w}_{i}\left(\pi^{*}(\phi) w\right) .
$$

Using (3-1), the facts that $w, \widehat{w}_{1}, \widehat{w}_{2}$ are Whittaker functionals, and by changing variables, we observe for all $z \in \bar{Z}$ that

$$
R_{i}(\lambda(z) \phi)=\psi^{-1}(z) R_{i}(\phi), \quad R_{i}(\rho(z) \phi)=\psi(z) R_{i}(\phi) .
$$

From Theorem 3.6 it follows that $R_{i}(\phi)=R_{i}\left({ }^{\bar{\tau}} \phi\right)$. Hence,

$$
\widehat{w}_{i}\left(\hat{\pi}(h) \pi^{*}(\phi) w\right)=\widehat{w}_{i}\left(\pi^{*}\left({ }^{\bar{\tau}}(\lambda(h) \phi) w\right)\right) .
$$

Using a change of variables again, we also have

$$
\pi^{*}\left({ }^{\bar{\tau}}(\lambda(h) \phi)\right) w=\pi^{*}\left({ }^{\bar{\tau}} \phi\right)\left(\pi^{*}\left({ }^{\bar{\tau}} h\right) w\right) .
$$

Joining (3-2) and (3-3) we obtain

$$
\widehat{w}_{i}\left(\hat{\pi}(h) \pi^{*}(\phi) w\right)=\widehat{w}_{i}\left(\pi^{*}\left({ }^{\bar{\tau}} \phi\right)\left(\pi^{*}\left({ }^{\bar{\tau}} h\right) w\right)\right) .
$$

In particular, if $\pi^{*}(\phi) w$ is the zero functional for some $\phi \in S\left(\overline{\operatorname{Sp}_{2 n}(F)}\right)$, then, for all $h \in \overline{\operatorname{Sp}_{2 n}(F)}, \widehat{w}_{i}\left(\pi^{*}\left({ }^{\bar{\tau}} \phi\right)\left(\pi^{*}\left({ }^{\bar{\tau}} h\right) w\right)\right)=0$. Here, for all $f \in S\left(\overline{\operatorname{Sp}_{2 n}(F)}\right)$, we have 


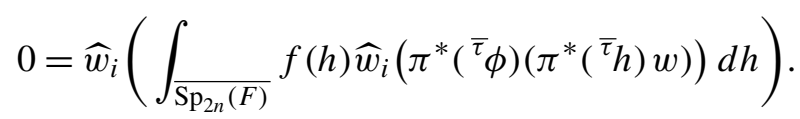

By the definition of $\pi^{*}(\phi) w$ and by changing the order of integration, we have, for all $v \in V_{\pi}$,

$$
\int_{\overline{\mathrm{Sp}}_{2 n}(F)} f(h) \widehat{w}_{i}\left(\pi^{*}\left({ }^{\bar{\tau}} \phi\right)\left(\pi^{*}\left({ }^{\bar{\tau}} h\right) w\right)\right)(v) d h=\hat{\pi}\left({ }^{\bar{\tau}} \phi\right)\left(\pi^{*}(f) w\right)(v) .
$$

The last two equalities yield that if $\pi^{*}(\phi) w=0$, then, for all $v \in V_{\pi}$ and $f \in$ $S\left(\overline{\operatorname{Sp}_{2 n}(F)}\right)$,

$$
\widehat{w}_{i}\left(\hat{\pi}\left({ }^{\bar{\tau}} \phi\right)\left(\pi^{*}(f) w\right)\right)(v)=0 .
$$

Because $\pi$ is irreducible one can conclude that

$$
\pi^{*}\left(S\left(\overline{\operatorname{Sp}_{2 n}(F)}\right) w\right)=V_{\hat{\pi}} .
$$

Indeed, since $\pi^{*}\left(S\left(\overline{\operatorname{Sp}_{2 n}(F)}\right) w\right)$ is an $\overline{\operatorname{Sp}_{2 n}(F)}$, invariant subspace we only have to show that $\pi^{*}\left(S\left(\overline{\operatorname{Sp}_{2 n}(F)}\right) w\right) \neq\{0\}$, which is clear.

Hence, changing variables once more, we see that for all $\xi \in V_{\hat{\pi}}$ we have

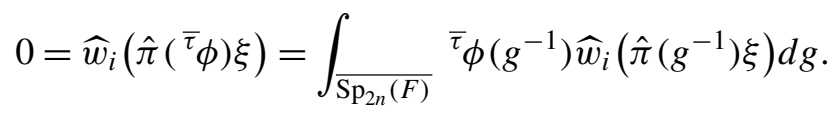

For $g \in \overline{\mathrm{Sp}_{2 n}(F)}$, define ${ }^{\omega} g=\bar{\tau}^{-1}$, and for $\phi \in S\left(\overline{\operatorname{Sp}_{2 n}(F)}\right)$, define ${ }^{\omega} \phi(g)=$ $\phi\left({ }^{\omega} g\right)$. We have just seen that if $\pi^{*}(\phi) w=0$ then

$$
\left((\hat{\pi})^{*}\left({ }^{\omega} \phi\right)\right) \widehat{w}_{i}=0 .
$$

This and the equality $\pi^{*}\left(S\left(\overline{\operatorname{Sp}_{2 n}(F)}\right) w\right)=V_{\hat{\pi}}$ show the following linear maps are well defined. For $i=1,2$, define $S_{i}: V_{\hat{\pi}} \rightarrow V_{\hat{\pi}}$ by $S_{i}\left(\pi^{*}(\phi) w\right)=\left((\hat{\pi})^{*}\left({ }^{\omega} \phi\right)\right) \widehat{w}_{i}$. One can easily check that $S_{1}$ and $S_{2}$ are two intertwining maps from $\hat{\pi}$ to $h \mapsto$ $\widehat{\hat{\pi}}\left(\sigma_{0}{ }^{t} h^{-1} \sigma_{0}\right)$. The last representation is clearly isomorphic to $h \mapsto \pi\left(\sigma_{0}{ }^{t} h^{-1} \sigma_{0}\right)$, which, due to the irreducibility of $\pi$, is itself irreducible. Schur's Lemma guarantees now the existence of a complex number $c$ such that $S_{2}=c S_{1}$. Thus, for all $\phi \in S\left(\overline{\operatorname{Sp}_{2 n}(F)}\right)$ and for all $\xi \in V_{\hat{\pi}}$, we have

$$
\int_{\overline{\mathrm{Sp}_{2 n}(F)}} \phi(g)\left(\widehat{w}_{2}-c \widehat{w}_{1}\right)\left(\hat{\pi}\left(g^{-1}\right) \xi\right) d g=0 .
$$

We can now conclude that $\widehat{w}_{1}$ and $\widehat{w}_{2}$ are proportional.

3B. Proof of Theorem 3.6. Put $G=\overline{\mathrm{Sp}_{2 n}(F)}$ and define $H=\bar{Z} \times \bar{Z}$. Let $\widetilde{\psi}$ be the character of $H$ defined by $\widetilde{\psi}\left(n_{1}, n_{2}\right)=\psi\left(n_{1}^{-1} n_{2}\right) . H$ acts on $G$ by $\left(n_{1}, n_{2}\right)$. $g=n_{1} g n_{2}^{-1}$. For $g \in G$, we denote by $H_{g}$ the stabilizer of $g$ in $H$. It is clearly a unimodular group. Let $Y$ be an $H$ orbit, that is, a subset of $G$ of the form 
$H \cdot g=Z g Z$, where $g$ is a fixed element in $G$. Let $S(Y)$ be the space of Schwartz functions on $Y$. $H$ acts on $S(Y)$ by

$$
(h \cdot \phi)(k)=\phi\left(h^{-1} \cdot k\right) \widetilde{\psi}^{-1}(h) .
$$

With this notation, the proof of Theorem 3.6 goes almost word for word as [Soudry 1987, proof of Theorem 2.3]. The main ingredient of that proof was [Bernstein and Zelevinskii 1976, Theorem 6.10], which asserts that the following four conditions imply Theorem 3.6:

(i) The set $\{(g, h \cdot g) \mid g \in G, h \in H\}$ is a union of finitely many locally closed subsets of $G \times G$.

(ii) For each $h \in H$, there exists $h_{\bar{\tau}} \in H$ such that $h \cdot{ }^{\bar{\tau}} g={ }^{\bar{\tau}}\left(h_{\bar{\tau}} \cdot g\right)$ for all $g \in G$.

(iii) $\bar{\tau}$ is of order 2.

(iv) Let $Y$ be an $H$ orbit. Suppose that there exists a non zero functional on $S(Y)$ satisfying $D(h \cdot \phi)=D(\phi)$ for all $\phi \in S(Y)$ and $h \in H$. Then ${ }^{\bar{\tau}} Y=Y$ and $D\left({ }^{\bar{\tau}} \phi\right)=D(\phi)$ for all $\phi \in S(Y)$.

Of these four conditions, only the forth requires some work. To make Soudry's proof work in our context, we have only to change [Soudry 1987, Theorem 2.2] to

Theorem 3.7. Fix $g \in G$. If for all $h \in H_{g}$, we have $\widetilde{\psi}^{-1}(h)=1$, then there exists an $h^{g} \in H$ such that $h^{g} \cdot g={ }^{\bar{\tau}} g$ and $\widetilde{\psi}^{-1}(h)=1$.

Before we prove this theorem, we state and prove its analog for $\operatorname{Sp}_{2 n}(F)$. After the proof, we give a short argument which completes the proof of Theorem 3.7.

Lemma 3.8. For a fixed $g \in \mathrm{Sp}_{2 n}(F)$ one of the following holds

A. There exist $n_{1}, n_{2} \in Z$ such that $n_{1} g n_{2}=g$ and $\psi\left(n_{1} n_{2}\right) \neq 1$

B. There exist $n_{1}, n_{2} \in Z$ such that $n_{1} g n_{2}={ }^{\tau} g$ and $\psi\left(n_{1} n_{2}\right)=1$.

Proof. Because $\tau$ preserves both $Z$ and $\psi$, it is enough to prove this Lemma only for a complete set of representatives of $Z \backslash \mathrm{Sp}_{2 n}(F) / Z$. We recall the Bruhat decomposition: $\operatorname{Sp}_{2 n}(F)=\bigcup_{w \in W} Z T w Z$, where $W$ is the Weyl group of $\operatorname{Sp}_{2 n}(F)$ and $T$ is the group of diagonal elements in $\operatorname{Sp}_{2 n}(F)$. We realize the set of Weyl elements as $\left\{\bar{w}_{\sigma} \tau_{S} \mid \sigma \in S_{n}, S \subseteq\{1,2, \ldots, n\}\right\}$, where, for $\sigma \in S_{n}$, we define $w_{\sigma} \in \mathrm{GL}_{n}(F)$ by $\left(w_{\sigma}\right)_{i, j}=\delta_{i, \sigma(j)}$ and $\bar{w}_{\sigma} \in \operatorname{Sp}_{2 n}(F)$ by

$$
\bar{w}_{\sigma}=\left(\begin{array}{cc}
w_{\sigma} & 0 \\
0 & w_{\sigma}
\end{array}\right) \text {. }
$$

Thus we may take $\left\{d^{-1} \bar{w}_{\sigma}^{-1} \varphi_{S} \mid d \in T, \sigma \in S_{n}, S \subseteq\{1,2, \ldots, n\}\right\}$ as a complete set of representatives of $Z \backslash \mathrm{Sp}_{2 n}(F) / Z$, where

$$
\varphi_{S}=\tau_{S^{c}} a_{S^{c}}=\left(\begin{array}{cc}
M_{S} & M_{S^{c}} \\
-M_{S^{c}} & M_{S}
\end{array}\right)
$$


and where, for $S \subseteq\{1,2, \ldots, n\}, M_{S} \in \operatorname{Mat}_{n \times n}(F)$ has $\left(M_{S}\right)_{i, j}=\delta_{i, j} \delta_{i \in S}$.

Denote by $w_{k}$ the $k \times k$ invertible matrix with elements $\left(w_{k}\right)_{i, j}=\delta_{i+j, k+1}$. Suppose that $k_{1}, k_{2}, \ldots, k_{p}, k$ are non negative integers such that $k+\sum_{i=1}^{p} k_{i}=n$. Suppose also that $a_{1}, a_{2}, \ldots, a_{p} \in F^{*}$ and $\eta \in\{ \pm 1\}$. For

$$
\begin{aligned}
\bar{w}_{\sigma} & =\operatorname{diag}\left(w_{k_{1}}, w_{k_{2}}, \ldots, w_{k_{p}}, I_{k}, w_{k_{1}}, w_{k_{2}}, \ldots, w_{k_{p}}, I_{k}\right), \\
d & =\operatorname{diag}\left(a_{1} \epsilon_{k_{1}}, a_{2} \epsilon_{k_{2}}, \ldots, a_{p} \epsilon_{k_{p}}, \eta I_{k}, a_{1}^{-1} \epsilon_{k_{1}}, a_{2}^{-1} \epsilon_{k_{2}}, \ldots, a_{p}^{-1} \epsilon_{k_{p}}, \eta I_{k}\right),
\end{aligned}
$$

and $S=\{n-k+1, n-k, \ldots, n\}$, one checks that

$$
{ }^{\tau}\left(d^{-1} \bar{w}_{\sigma}^{-1} \varphi_{S}\right)=d^{-1} \bar{w}_{\sigma}^{-1} \varphi_{S}
$$

Thus $d^{-1} \bar{w}_{\sigma}^{-1} \varphi_{S}$ is of type $B$.

We shall show that all other representatives are of type $A$. We will prove that in all the other cases one can find $n_{1}, n_{2} \in Z$ such that

$$
\begin{gathered}
\bar{w}_{\sigma} d n_{1} d^{-1} \bar{w}_{\sigma}^{-1}=\varphi_{S} n_{2}^{-1} \varphi_{S}^{-1}, \\
\psi\left(n_{1} n_{2}\right) \neq 1 .
\end{gathered}
$$

We shall use the following notations and facts: Denote by $E_{p, q}$ the $n \times n$ matrix defined by $\left(E_{p, q}\right)_{i, j}=\delta_{p, i} \delta_{q, j}$. For $i, j \in\{1,2, \ldots, n\}, i \neq j$ we define the root subgroups of $\mathrm{Sp}_{2 n}(F)$ through

$$
\begin{aligned}
U_{i, j} & =\left\{u_{i, j}(x)=\left(\begin{array}{cc}
I_{n}+x E_{i, j} & 0 \\
0 & I_{n}-x E_{j, i}
\end{array}\right) \mid x \in F\right\} \simeq F, \\
V_{i, j} & =\left\{v_{i, j}(x)=\left(\begin{array}{cc}
I_{n} & x E_{i, j}+x E_{j, i} \\
0 & I_{n}
\end{array}\right) \mid x \in F\right\} \simeq F, \\
V_{i, i} & =\left\{v_{i, i}(x)=\left(\begin{array}{cc}
I_{n} & x E_{i, i} \\
0 & I_{n}
\end{array}\right) \mid x \in F\right\} \simeq F .
\end{aligned}
$$

If $i<j$, we call $U_{i, j}$ a positive root subgroup; if $j=i+1$, we call $U_{i, j}$ a simple root subgroup; and if $j>i+1$, we call $U_{i, j}$ a nonsimple root subgroup. We call ${ }^{\mathrm{t}} U_{i, j}=U_{j, i}$ the negative of $U_{i, j}$. The group $S_{n}$ acts on the set $\left\{U_{i, j} \mid i, j \in\right.$ $\{1,2, \ldots, n\}, i \neq j\}$ by

$$
\bar{w}_{\sigma} u_{i, j}(x) \bar{w}_{\sigma}^{-1}=u_{\sigma(i), \sigma(j)}(x)
$$

and on the set $\left\{V_{i, j} \mid i, j \in\{1,2, \ldots, n\}\right\}$ by

$$
\bar{w}_{\sigma} v_{i, j}(x) \bar{w}_{\sigma}^{-1}=v_{\sigma(i), \sigma(j)}(x) .
$$


$T$ acts on each root subgroup via rational characters:

$$
\begin{aligned}
d u_{i, j}(x) d^{-1} & =u_{i, j}\left(x d_{i} d_{j}^{-1}\right), \\
d v_{i, j}(x) d^{-1} & =v_{i, j}\left(x d_{i} d_{j}\right),
\end{aligned}
$$

where $d=\operatorname{diag}\left(d_{1}, d_{2}, \ldots, d_{n}, d_{1}^{-1}, d_{2}^{-1}, \ldots, d_{n}^{-1}\right)$. We also note that

$$
\begin{array}{ll}
\varphi_{S} v_{i, i}(x) \varphi_{S}^{-1}=v_{i, i}(x) & \text { if } i \in S, \\
\varphi_{S} v_{i, j}(x) \varphi_{S}^{-1}=u_{i, j}(x) & \text { if } i \in S, j \notin S, \\
\varphi_{S} u_{i, j}(x) \varphi_{S}^{-1}=u_{i, j}(x) & \text { if } i \in S, j \in S, i \neq j, \\
\varphi_{S} u_{i, j}(x) \varphi_{S}^{-1}=u_{j, i}(-x)={ }^{\mathrm{t}} u_{i, j}(x)^{-1} & \text { if } i \notin S, j \notin S, i \neq j .
\end{array}
$$

Consider the representative $d^{-1} \bar{w}_{\sigma}^{-1} \varphi_{S}$. Assume first that $S$ is empty. If there exists a simple root subgroup $U_{k, k+1}$ taken by $\sigma$ to the negative of a nonsimple root subgroup, we choose $n_{1}=u_{k, k+1}(x)$ and $n_{2}={ }^{\mathrm{t}} u_{\sigma(k), \sigma(k+1)}\left(d_{k} d_{k+1}^{-1} x\right)$. For such a choice, by (3-8), (3-10) and (3-15), Equation (3-6) holds. Also, since $\psi\left(n_{1} n_{2}\right)=$ $\psi\left(n_{1}\right)=\psi(x)$, it is possible, by choosing $x$ properly, to satisfy (3-7). Next, if there exists a nonsimple positive root subgroup $U_{i, j}$ taken by $\sigma$ to the negative of a simple root subgroup, we choose $n_{1}=u_{i, j}(x)$ and $n_{2}={ }^{\mathrm{t}} u_{\sigma(i), \sigma(j)}\left(d_{i} d_{j}^{-1} x\right)$ and repeat the argument. Thus, $d^{-1} \bar{w}_{\sigma}^{-1} \varphi_{\varnothing}$ is of type A unless $\sigma$ has the following two properties: first, if $\sigma$ takes a simple root to a negative root, then it is taken to the negative of a simple root; second, if $\sigma$ takes a nonsimple positive root to a negative root, then it is taken to the negative of a nonsimple root. An easy argument shows that if $\sigma$ has these two properties, $\bar{w}_{\sigma}$ must be as in (3-4) with $k=0$. We assume now that $\bar{w}_{\sigma}$ has this form. To finish the case $S=\varnothing$, we show that unless $d$ has the form (3-5), with $k=0$ and $k_{1}, k_{2}, \ldots, k_{p}$ corresponding to $\bar{w}_{\sigma}$, then $d^{-1} \bar{w}_{\sigma}^{-1} \varphi_{\varnothing}$ is of type A. Indeed, suppose that there exist $d_{k}$ and $d_{k+1}$ that belong to the same block in $\bar{w}_{\sigma}$ such that $d_{k} \neq-d_{k+1}$. Then we choose $n_{1}=u_{k, k+1}(x)$ and $n_{2}={ }^{\mathrm{t}} u_{\sigma(k), \sigma(k+1)}\left(d_{k} d_{k+1}^{-1} x\right)$. For such a choice (3-6) holds as before, and $\psi\left(n_{1} n_{2}\right)=\psi\left(x\left(1+d_{k} d_{k+1}^{-1}\right)\right)$. Hence it is possible to choose $x$ so (3-7) holds.

We may now assume $|S| \geq 1$. We show that if $n \notin S$ then $d^{-1} \bar{w}_{\sigma}^{-1} \varphi_{S}$ is of type A. Indeed, if $\sigma(n) \in S$ (so $\sigma(n) \neq n$ ), then, for all $x \in F$, if we choose $n_{1}=v_{n, n}(x)$ and $n_{2}=v_{\sigma(n), \sigma(n)}\left(-x d_{n}^{2}\right)$, then (3-9), (3-10), and (3-12) imply Equation (3-6) holds. Clearly, there exists $x \in F$ such that $\psi\left(n_{1} n_{2}\right)=\psi\left(n_{1}\right)=\psi(x) \neq 1$. Suppose now that $n \notin S \neq \varnothing$ and $\sigma(n) \notin S$. In this case, we can find $1 \leq k \leq n-1$ such that

$$
\sigma(k) \in S \quad \text { and } \quad \sigma(k+1) \notin S .
$$

We choose

$$
n_{1}=u_{k, k+1}(x) \quad \text { and } \quad n_{2}=v_{\sigma(k), \sigma(k+1)}\left(-x d_{k} d_{k+1}^{-1}\right)
$$


By (3-8), (3-10) and (3-13), Equation (3-6) holds, and since $\psi\left(n_{1} n_{2}\right)=\psi\left(n_{1}\right)=$ $\psi(x)$, we can satisfy (3-7) by properly choosing $x$. We assume now $n \in S$. We also assume $\sigma(n) \in S$, and otherwise we use the last $\operatorname{argument}$. Fix $n_{1}=v_{n, n}(x)$ and $n_{2}=v_{\sigma(n), \sigma(n)}\left(-x d_{n}^{2}\right)$. One can check using (3-9), (3-11), and (3-12) that (3-6) holds. Note that

$$
\psi\left(n_{1} n_{2}\right)= \begin{cases}x, & \sigma(n) \neq n, \\ x\left(d_{n}^{2}-1\right), & \sigma(n)=n .\end{cases}
$$

Hence, $d^{-1} \bar{w}_{\sigma}^{-1} \varphi_{S}$ is of type A unless $\sigma(n)=n$ and $d_{n}= \pm 1$, and so we now assume that of $\sigma(n)$ and $d_{n}$. If $S=\{n\}$, we use a similar argument to the one we used for $S=\varnothing$ but this time analyze the action of $\sigma$ on $\{1,2,3, \ldots, n-1\}$.

We are left with the case $\sigma(n)=n, d_{n}= \pm 1$, and $S \supsetneq\{n\}$. If $\sigma(n-1) \notin S$ we repeat an argument we used already. We choose $1 \leq k \leq n-2, n_{1}$, and $n_{2}$ as in (3-16) and (3-17). We now assume $\sigma(n-1) \in S$. We choose $n_{1}=u_{n-1, n}(x)$ and $n_{2}=u_{\sigma(n-1), n}\left(-x d_{n-1} d_{n}^{-1}\right)$. Using (3-8), (3-10) and (3-14), we observe that (3-6) holds. Also

$$
\psi\left(n_{1} n_{2}\right)= \begin{cases}x, & \sigma(n-1) \neq n-1 \\ x\left(d_{n-1} d_{n}^{-1}-1\right), & \sigma(n-1)=n-1\end{cases}
$$

Thus, unless $\sigma(n-1)=n-1$ and $d_{n-1}=d_{n}= \pm 1, d^{-1} \bar{w}_{\sigma}^{-1} \varphi_{S}$ is of type A. Therefore, we should only consider the case $\sigma(n)=n, \sigma(n-1)=n-1, d_{n-1}=$ $d_{n}= \pm 1$, and $\{n-1, n\} \subseteq S$. We continue in the same course. If $S=\{n-$ $1, n\}$, we use similar argument we used for $S=\varnothing$, analyzing the action of $\sigma$ on $\{1,2,3, \ldots, n-2\}$. If $S \supsetneq\{n-1, n\}$, we show that unless $\sigma(n-2)=n-2 \in S$ and $d_{n-2}=d_{n-1}=d_{n}= \pm 1$ we are in type A etc.

We now complete the proof of Theorem 3.7. We define types $\bar{A}$ and $\bar{B}$ for $\overline{\operatorname{Sp}_{2 n}(F)}$ by analogy with the definitions given in Lemma 3.8 and show that each element of $\bar{Z} \backslash \overline{\mathrm{Sp}_{2 n}(F)} / \bar{Z}$ is either of type $\bar{A}$ or of type $\bar{B}$. Given $\bar{g}=(g, \epsilon) \in$ $\overline{\mathrm{Sp}_{2 n}(F)}$, if $g$ is of type $\mathrm{A}$, then there are $n_{1}, n_{2} \in Z$ such that $n_{1} g n_{2}=g$ and $\psi\left(n_{1} n_{2}\right) \neq 1$. Let $\bar{n}_{i}=\left(n_{i}, 1\right)$. Clearly $\bar{n}_{1} \bar{g} \bar{n}_{2}=\bar{g}$ and $\psi\left(\bar{n}_{1} \bar{n}_{2}\right)=\psi\left(n_{1} n_{2}\right) \neq 1$. If $g$ is not of type A, then by Lemma 3.8 it is of type B. There are $n_{1}, n_{2} \in Z$ such that $n_{1} g n_{2}={ }^{\tau} g$ and $\psi\left(n_{1} n_{2}\right)=1$. Define $\bar{n}_{i}$ as before. Note that $\psi\left(\bar{n}_{1} \bar{n}_{2}\right)=$ $\psi\left(n_{1} n_{2}\right)=1$. From Lemma 3.5 it follows that $\bar{n}_{1} \bar{g} \bar{n}_{2}={ }^{\bar{\tau}} \bar{g}$. This proves Lemma 3.8 for $\overline{\mathrm{Sp}_{2 n}(F)}$, which is Theorem 3.7.

\section{Acknowledgment}

I would like to thank David Soudry for endless hours of conversation. Without his devoted help this work would not have been done. 


\section{References}

[Banks 1998] W. D. Banks, "Heredity of Whittaker models on the metaplectic group", Pacific J. Math. 185:1 (1998), 89-96. MR 99m:22016 Zbl 0980.22021

[Barthel 1991] L. Barthel, "Local Howe correspondence for groups of similitudes", J. Reine Angew. Math. 414 (1991), 207-220. MR 92g:22037 Zbl 0708.22006

[Bernstein and Zelevinskii 1976] I. Bernstein and A. Zelevinskii, "Representations of the group $\operatorname{GL}(n, F)$ where $F$ is a non-archimedean local field", Russian Math. Surv. 31:3 (1976), 1-68. Zbl 0348.43007

[Bump et al. 1991] D. Bump, S. Friedberg, and J. Hoffstein, " $p$-adic Whittaker functions on the metaplectic group”, Duke Math. J. 63:2 (1991), 379-397. MR 92d:22024 Zbl 0758.22009

[Gelbart 1976] S. S. Gelbart, Weil's representation and the spectrum of the metaplectic group, Springer, Berlin, 1976. Lecture Notes in Mathematics, Vol. 530. MR 54 \#12654 Zbl 0365.22017

[Gelbart et al. 1979] S. Gelbart, R. Howe, and I. Piatetski-Shapiro, "Uniqueness and existence of Whittaker models for the metaplectic group”, Israel J. Math. 34:1-2 (1979), 21-37. MR 81j:22021 Zbl 0441.22015

[Gel'fand and Kazhdan 1975] I. M. Gel'fand and D. A. Kazhdan, "Representations of the group $\mathrm{GL}(n, K)$ where $K$ is a local field", pp. 95-118 in Lie groups and their representations (Budapest, 1971), edited by I. M. Gel'fand, Halsted, New York, 1975. MR 53 \#8334 Zbl 0348.22011

[Kubota 1969] T. Kubota, On automorphic functions and the reciprocity law in a number field, Lectures in Mathematics, Department of Mathematics, Kyoto University, No. 2, Kinokuniya BookStore Co. Ltd., Tokyo, 1969. MR 41 \#152 Zbl 0231.10017

[Kudla 1994] S. S. Kudla, "Splitting metaplectic covers of dual reductive pairs", Israel J. Math. 87:1-3 (1994), 361-401. MR 95h:22019 Zbl 0840.22029

[Mœglin et al. 1987] C. Mœglin, M.-F. Vignéras, and J.-L. Waldspurger, Correspondances de Howe sur un corps p-adique, Lecture Notes in Mathematics 1291, Springer, Berlin, 1987. MR 91f:11040 Zbl 0642.22002

[Rao 1993] R. R. Rao, "On some explicit formulas in the theory of Weil representation", Pacific J. Math. 157:2 (1993), 335-371. MR 94a:22037 Zbl 0794.58017

[Rodier 1973] F. Rodier, "Whittaker models for admissible representations of reductive $p$-adic split groups", pp. 425-430 in Harmonic analysis on homogeneous spaces (Williamstown, MA, 1972), vol. XXVI, edited by C. C. Moore, Amer. Math. Soc., Providence, R.I., 1973. MR 50 \#7419 Zbl 0287.22016

[Shalika 1974] J. A. Shalika, "The multiplicity one theorem for GL ", Ann. of Math. (2) 100 (1974), 171-193. MR 50 \#545 Zbl 0316.12010

[Soudry 1987] D. Soudry, "A uniqueness theorem for representations of GSO(6) and the strong multiplicity one theorem for generic representations of GSp(4)", Israel J. Math. 58:3 (1987), 257287. MR 89f:22025 Zbl 0642.22003

Received May 22, 2006.

DANI SZPRUCH

SCHOOL OF Mathematical SCIENCES

The RAYMOND AND BEVERLy SACKLER FACUlty OF EXACT SCIENCES

TEL AVIV UNIVERSITY

TEL AVIV 69978

ISRAEL

szpruchd@zahav.net.il 